\title{
Correction to: The peptide mimicking small extracellular ring domain of CD82 inhibits tumor cell migration in vitro and metastasis in vivo
}



Published online: 5 May 2021

○) Springer-Verlag GmbH Germany, part of Springer Nature 2021

\section{Correction to: Journal of Cancer Research and Clinical Oncology https://doi.org/10.1007/s00432-021-03595-6}

In the original article published, the three figures are cited incorrectly under the sub-headings.

Under the title "CD82EC1-mP inhibits cancer cell adhesion" the figure is cited incorrectly as $2 \mathrm{~b}$. The correct figure name is 4.

Under the title "CD82EC1-mP abrogates the lung metastases in animal models" the figure is cited incorrectly as Fig. 3 and Fig. 4. The correct figure name is 5 and 6.

The original article has been corrected.
The original article can be found online at https://doi.org/10.1007/ s00432-021-03595-6.

\section{Xiaohua Huang}

xiaohua312@sina.com

\section{Keli Ma}

makelipaper@163.com

$\mathrm{Xin} \mathrm{He}$

fly.poxiao@qq.com

Xiaoguang Ma

afei_007_2001@sina.com

Congcong Wang

292629810@qq.com

Mingchun Luan

yjslmc@163.com
Publisher's Note Springer Nature remains neutral with regard to jurisdictional claims in published maps and institutional affiliations.

Ying Li

dyeyly@163.com

1 Department of Biochemistry and Molecular Biology, Dalian Medical University, Dalian 116044, China

2 Department of Respirotory and Clinical Medecine, First Affiliated Hospital of Dalian Medical University, Dalian, China

3 Department of Clinical Laboratory, Second Affiliated Hospital of Dalian Medical University, Dalian, China

4 Department of Clinical Biochemistry, College of Laboratory Medicine, Dalian Medical University, Dalian, China 